\title{
Controversies relating to preparticipation cardiovascular screening in young athletes: time for a realistic solution?
}

\author{
M Papadakis, ${ }^{1,2}$ N Chandra, ${ }^{1,2}$ S Sharma ${ }^{1,2}$
}

The sudden death of any young individual is a tragic event that causes immeasurable damage to family lives. The sudden death of a young athlete from a cardiac disorder is particularly emotive and is often associated with considerable media coverage, drawing attention to the youth, the athletic prowess of the individual and the number of life years lost consequent to a cardiac disorder that could have been detected during life. Most exercise-related sudden cardiac deaths (SCDs) are attributed to congenital or hereditary cardiac disorders that are asymptomatic in most victims. ${ }^{1}{ }^{2}$ Unsurprisingly, the death of a young athlete often galvanises urgent discussions relating to preparticipation cardiac screening involving members of the community, sports physicians and sporting governing bodies.

There is considerable resistance to implementing widespread cardiac screening of athletes. The low incidence of deaths and the low prevalence rates of all implicated disorders challenge the cost efficacy of such a programme. Furthermore, there are concerns relating to the overlap between the physiological adaptation to exercise and the cardiac disease resulting in falsepositive results and unnecessary anxiety or even disqualification of an athlete from future competitive sport. ${ }^{3}$ It is also recognised that up to $11 \%$ of deaths in athletes occur at rest; therefore, cessation of sport will not necessarily prevent death in all athletes. ${ }^{1}$ Finally, there are issues relating to the lack of infrastructure and expert personnel trained in athlete's heart and the broad phenotype of the heterogeneous disorders implicated in the sudden death of an athlete. The UK and many other Western countries do not favour

${ }^{1}$ King's College Hospital, London, UK; ' University Hospital Lewisham, London, UK

Correspondence to Professor Sanjay Sharma, King's College Hospital, Denmark Hill, London SE5 9RS, UK; ssharma21@hotmail.com screening of athletes. Indeed, screening of athletes in the UK is confined to elite sporting organisations such as the Premier League football association and the Lawn Tennis Association that mandate independently financed screening programmes in all youth athletes.

In the USA and Italy, preparticipation cardiac screening programmes are in existence to minimise the risk of sudden death due to cardiac disorders in young athletes. ${ }^{4}$ The US programme utilises a health questionnaire relating to cardiac symptoms and a family history of premature cardiac disease, as well as physical examination of the cardiovascular system. Unfortunately, the reputation of such basic screening programmes has been jeopardised by American physicians holding international expert status in sports cardiology studies that have emphatically demonstrated an extremely poor yield in identifying athletes with fatal disorders. ${ }^{5}$

In Italy, a state-sponsored screening programme has been in place since the late 1970s and includes a health questionnaire, a cardiovascular physical examination and a 12-lead electrocardiography (ECG). The Italian experience from Veneto has shown that screening with ECG has reduced the death rate in athletes from 4.19/100 000 person-years to $0.43 / 100000$ person-years, equating to a reduction of approximately $90 \% .^{6}$ The data have received immense publicity and are considered the criterion standard paradigm for low cost and effective cardiac screening in young athletes. The impact of the number of life years saved is laudable and would be considered a health service boost if similar number of life years were preserved in individuals in the fifth decade onwards. The reduction in these deaths was associated with a $7 \%$ of individuals requiring further investigations because of false-positive test results and the disqualification of $2 \%$ of all athletes screened predominantly because of hypertension, cardiac conduction tissue disease and valvular heart disease. Most disqualifications are on anecdotal grounds and lack evidence-based credibility.

Unfortunately, there are no data from other European countries in the literature relating to screening young athletes that are comparable in magnitude with the Italian data, which can be simply explained by the fact that screening competitive athletes is not mandatory in most countries. Elston and Stein extrapolate findings from the Italian experience to apply directly to the UK in an attempt to facilitate the debate regarding the benefits, but more importantly, the potential harms of screening. Based on this fundamental assumption, the numerical implications of false-positive results and expected disqualifications from sport are discouraging. Of the potential 1520021 young athletes screened, 140361 would require further investigations, and for every life saved, 791 athletes would be disqualified. ${ }^{4}$

However, the epidemiological study by Elston and Stein exhibits important limitations that may have influenced the results and underestimated the impact of screening in the prevention of SCDs in athletes and the population as a whole. The authors consider as postscreening period the first 23 years of screening (1982-2004), claiming that this may provide a more realistic picture, as it includes the period when screening mechanisms and expertise were developed and the full potential of screening is unlikely to have been realised. Consequently, the reported postscreening incidence of SCD is quoted as 1.57/100 000 person-years. This estimate is twofold higher than the reported incidence by Corrado et al of $0.87 / 100000$ person-years during the late screening period (1993-2004) and fourfold higher than the reported incidence of $0.43 / 100000$ person-years in the 2001-2004 period. Although a longer period may offer epidemiologically more robust results, it is important to acknowledge that the latter figure of $0.43 / 100000$ person-years is likely to provide a more accurate estimate of the real potential of screening that is reinforced by the fact that the incidence of SCD plateaus during the 2001-2004 period and is likely to have remained so during the proceeding years.

Furthermore, Elston and Stein underestimate the incidence of SCD in young (14-35 years old) individuals in the UK. A recent study analysing the Office of National Statistics data indicates around 400 SCDs per year in the UK in this age group, which is likely to be 
an underestimate of the true incidence given the experience from previous prospective epidemiological studies within the UK and potential misclassifications of SCDs as epilepsy or accidental drowning.?

The study also fails to take into account current screening experience in the UK. ${ }^{8}$ Our own experience of screening highly trained athletes using the Italian model indicates a lower false-positive rate of $3.7 \%$, compared with $9 \%$ by Corrado et al. This is most likely to reflect the differences in interpretation of an abnormal ECG because in our practice, isolated large ORS complexes, borderline right axis deviation, a borderline prolonged $\mathrm{QTc}^{9}$ and $\mathrm{T}$-wave inversions in the right precordial leads in athletes $<16$ years old ${ }^{10}$ would not be indications for further investigations in asymptomatic athletes in the absence of a family history of premature cardiac disease or SCD. Similarly, there is no evidence that athletes with hypertension who continue to exercise are at risk of a fatality, and the significance of mitral valve prolapse and isolated ventricular extrasystoles in the aetiology of sudden death is debatable. Therefore, such athletes should not be considered to be at risk of sudden death and would not be advised to refrain from exercise. Conversely, the UK is more ethnically diverse than the Veneto region of Italy. In particular, there are significantly more athletes of Afro-Caribbean origin in the UK who are known to exhibit bizarre repolarisation electrical changes and may generate a significant number of falsepositive results.

In a prospective and ongoing pilot study involving $>8000$ athletes conducted by the charitable organisation Cardiac Risk in the Young (CRY), a potentially fatal disorder is identified in $0.3 \%$. This figure is not dissimilar to the Italian data. However, the false-positive rate in this study is $<4 \%$ (unpublished data). Should one consider treatable conditions such as the Wolff-Parkinson-White syndrome and right ventricular outflow tract ventricular tachycardia, then the permanent disqualification rates and the number of athletes disqualified for every life saved would be reduced dramatically. Although the precise false-negative rate is unknown, a mean 8-year follow-up had identified only one athlete with an SCD from an anomalous coronary artery disease, a disorder that cannot be identified with 12-lead ECG Such prospective screening programmes are crucial to solving the debate and providing the British perspective relating to the cardiac screening of asymptomatic young athletes.

Finally, Elston and Stein places particular emphasis on minor harms such as elevated anxiety caused by false-positive results. Studies from established screening programmes indicate that individuals with a false-positive result may experience considerable anxiety until further investigations provide reassurance, which highlight the need for prompt evaluation of athletes who fail the initial screening. However, an apprehensive approach over long-term psychological scarring of athletes with an initial false-positive result requiring further detailed investigations is not justified because evidence from the same studies indicate that in the context of a well-organised screening programme with expert psychological support, there is no significant long-term psychological burden. ${ }^{3}$

The financial constraints within the National Health Service; the priority in apportioning the budgets in reducing deaths from myocardial infarction, stroke and neoplastic disorders; the lack of expertise and the lack of robust cost-effectiveness studies means that a nationwide screening of all young individuals participating in competitive sports cannot be currently implemented. Benevolent pragmatists advocate raising the awareness of the risk of SCD in sport and screening only those with a family history of hereditary cardiac disorders or symptomatic manifestation of cardiac disease. Such a strategy, however, will deny most athletes from being screened and fail to identify up to $80 \%$ of athletes at risk of sudden death. These limitations should prompt the development of a collaborative scheme between the government, charity organisations such as CRY and sporting bodies to provide an initiative to offer cost-effective screening with 12-lead ECG for athletes who choose to be screened for their self-protection.

Funding SS, MP and NC work in the National Health Service. MP and NC are funded by a research grant from the charitable organisation Cardiac Risk in the Young (CRY) which support preparticipation screening in young athletes

Competing interests The charitable organisation Cardiac Risk in the Young (CRY), which supports preparticipation screening in young athletes, has provided facilities, including necessary staffing, electrocardiography and echocardiography machines for screening many national sporting squads including rugby, tennis, boxing, swimming, athletics and football. The data from the screening programme have resulted in several publications in major peer-reviewed journals. SS is a consultant cardiologist to CRY.

Patient consent Not obtained.

Contributors MP, NC and SS drafted the article and revised it critically for scientific content and approved the final version for publication. SS is the guarantor.

Provenance and peer review Not commissioned; not externally peer reviewed.

Accepted 18 September 2009

Published Online First 21 October 2009

Br J Sports Med 2011:45:165-166.

doi:10.1136/bjsm.2009.067652

\section{REFERENCES}

1. Corrado D, Basso C, Rizzoli G, et al. Does sports activity enhance the risk of sudden death in adolescents and young adults? J Am Coll Cardiol 2003;42:1959-63.

2. Maron BJ. Sudden death in young athletes. N Eng/ J Med 2003;349:1064-75.

3. Papadakis M, Sharma S. Electrocardiographic screening in athletes: the time is now for universal screening. Br J Sports Med 2009;43:663-8.

4. Elston J, Stein K. Public health implications of establishing a national programme to screen young athletes in the UK. Br J Sports Med Published Online First: 20 July 2009. doi:10.1136/bjsm.2009.061184.

5. Maron BJ, Shirani J, Poliac LC, et al. Sudden death in young competitive athletes. Clinical, demographic, and pathological profiles. JAMA 1996;276:199-204.

6. Corrado D, Basso C, Pavei A, et al. Trends in sudden cardiovascular death in young competitive athletes after implementation of a preparticipation screening program. JAMA 2006;296:1593-601.

7. Papadakis $\mathbf{M}$, Sharma S, Cox S, et al. The magnitude of sudden cardiac death in the young: a death certificate based review in England and Wales. Europace 2009;11:1353-8.

8. Wilson MG, Basavarajaiah S, Whyte GP, et al. Efficacy of personal symptom and family history questionnaires when screening for inherited cardiac pathologies: the role of electrocardiography. $\mathrm{Br} \mathrm{J}$ Sports Med 2008;42:207-11.

9. Basavarajaiah S, Wilson M, Whyte G, et al. Prevalence and significance of an isolated long QT interval in elite athletes. Eur Heart J 2007;28:2944-9.

10. Papadakis M, Basavarajaiah S, Rawlins J, et al. Prevalence and significance of T-wave inversions in 